Research Paper

\title{
CPA4 is a Novel Diagnostic and Prognostic Marker for Human Non-Small-Cell Lung Cancer
}

\author{
Lichao Sun ${ }^{1,2}$, Yipeng Wang ${ }^{3}$, Hebao Yuan², Joseph Burnett², Jian Pan ${ }^{4}$, Zhihua Yang1, Yuliang Ran ${ }^{1}$, Ila \\ Myers $^{2}$, Duxin Sun ${ }^{2}$ \\ 1. State Key Laboratory of Molecular Oncology, Cancer Hospital, Chinese Academy of Medical Sciences, Peking Union Medical College, Beijing, 100021, P. R. \\ China. \\ 2. Department of Pharmaceutical Sciences, University of Michigan, Ann Arbor, MI 48109. \\ 3. The Department of Abdominal Surgical Oncology Cancer Hospital, Chinese Academy of Medical Sciences (CAMS), Beijing 100021, P.R. China. \\ 4. Department of Hematology and Oncology, Children's Hospital of Soochow University, Suzhou, Jiangsu, 215005, P.R. China. \\ $\square$ Corresponding authors: Lichao Sun, PhD. State Key Laboratory of Molecular Oncology, Cancer Hospital, Chinese Academy of Medical Sciences, Peking \\ Union Medical College, Beijing, 100021, P. R. China. Prof_sunlichao@163.com Duxin Sun, PhD. Department of Pharmaceutical Sciences, University of Michigan, \\ Ann Arbor, MI 48109. duxins@umich.edu.
}

(0) Ivyspring International Publisher. Reproduction is permitted for personal, noncommercial use, provided that the article is in whole, unmodified, and properly cited. See http://ivyspring.com/terms for terms and conditions.

Received: 2016.02.05; Accepted: 2016.04.26; Published: 2016.06.18

\begin{abstract}
Background. Carboxypeptidase A4 (CPA4) belongs to a member of the metallocarboxypeptidase family, and its expression in lung cancer samples and clinical significance are still not investigated until now. In this study, we aimed to evaluate the level of CPA4 in non-small-cell lung cancer (NSCLC) samples and correlate its level with clinical outcome.

Methods. CPA4 gene expression in lung cancer tissues were analyzed by using the Oncomine database (www.oncomine.org). The expression of CPA4, Survivin and VEGF in lung cancer and adjacent normal tissues were evaluated by IHC using the corresponding primary antibodies on two different commercial tissue arrays (Shanghai Biochip Co., Ltd., Shanghai, China). Their levels in serum were determined by using commercial human enzyme-linked immunosorbent assay kits. We also examined their relations to clinicopathologic parameters, and explored the diagnostic and prognostic value in NSCLC.

Results. We identified an elevation of CPA4 in mRNA level and gene amplification in lung cancer tissues in comparison to normal lung tissues. High CPA4 expression was observed in 120/165 (72.7\%) NSCLC samples, and significantly correlated with Tumor size, Depth of invasion, Lymph Node Metastasis, Stage, VEGF level and Survivin level. High CPA4 expression is associated with poor prognosis of NSCLC patients. Multivariable Cox regression analysis demonstrated that CPA4 expression was an independent prognostic factor. Furthermore, serum CPA4 level was also significantly higher in NSCLC patients than in healthy controls. Logistic regression analysis revealed that serum CPA4 and CYFRA21-1 level were the significant parameters for detecting NSCLC. Receiver operating characteristic curves (ROC) in NSCLC patients versus normal people yielded the optimal cut-off value was $2.70 \mathrm{ng} / \mathrm{ml}$ for CPA4 and $19 \mathrm{ng} / \mathrm{ml}$ for CYFRA21-1, respectively. The area under ROC curve (AUC) was 0.830 for the combination of the two tumor markers.

Conclusion. Our results demonstrated that overexpression of CPA4 in NSCLC is associated with an unfavorable prognosis, and serum CPA4 level combining with serum CYFRA21-1 level could be used to aid early detection of NSCLC.
\end{abstract}

Key words: CPA4, lung Cancer, Marker, Prognosis, Diagnosis.

\section{Introduction}

Lung cancer is the leading cause of cancer-related death worldwide and non-small-cell lung cancer (NSCLC) accounts for approximately $80-85 \%$ of lung cancers [1]. Early detection and surgical resection of cancer confined to the primary site could significantly improve survival for lung cancer patients [2]. Currently, the widely used diagnostic markers for lung cancer are 
neuron-specific enolase (NSE), carcinoembryonic antigen (CEA), cytokeratin 19 fragment (CYFRA21-1) and squamous cell carcinoma antigen (SCC)[3]. However, these tumor markers in the diagnosis of lung cancer display relatively low sensitivity and specificity [4,5]. Thus, there is an urgent need to identify new biomarkers that can help to diagnose or monitor treatment for lung cancer.

Tumor angiogenesis is an essential step for tumor progression, which plays playing key roles in tumor invasion and metastasis [6]. And tumor cells can secrete factors that promote the neo-vascularisation. Among these growth factors, Survivin and vascular endothelial growth factor (VEGF) which have been reported to play important roles in formation of blood vessels $[7,8]$.

Carboxypeptidase A4 (CPA4) is a member of the metallocarboxypeptidase family [9].

Like other secreted proteins, CPA4 has been proved to be secreted from cells to catalyze the release of carboxy-terminal amino acids, which might help to establish a tumor micro-environment [10]. Although it has been reported that CPA4 was associated with prostate cancer aggressiveness, the clinical significance of CPA4 expression in NSCLC still remains unclear.

In this study, we firstly demonstrated that CPA4 level was significantly elevated in NSCLC tissues as well as serum samples, and was closely associated with poor prognosis of lung cancer. Furthermore, serum CPA4 might be a candidate diagnostic biomarker for NSCLC patients.

\section{Materials and methods}

\section{Analysis of Oncomine Data}

To determine the expression pattern of CPA4 in lung cancer, the datasets in Oncomine database (https://www.oncomine.org) were used. Briefly, CPA4 gene was queried in the database and the results were filtered by selecting lung cancer and Lung Cancer vs. Normal Analysis. The data were displayed by using Box chart. P-values for each group were calculated using student t-test. Details of standardized normalization techniques and statistical calculations are provided on the Oncomine.

\section{Clinical samples and Evaluation of Immunostaining}

Two different commercial tissue arrays were constructed by Shanghai Biochip Co. Ltd. as described [11]. One contains 90 cases of lung adenocarcinoma patients with matched adjacent normal tissues, and another contains 75 cases of Lung squamous cell carcinoma with matched adjacent normal tissues, respectively. For all the specimens, clinicopathological information (age, gender, pathology, differentiation, TNM stage, and follow-up data) was available. The expression of CPA4, VEGF and Survivin in the tissues was evaluated by immunohistochemical staining with specific antibodies. Standard Avidin-biotin complex peroxidase immunohistochemical staining was performed. Briefly, after deparaffinizationin xylene and graded alcohols, heated antigen retrieval was done in citrate buffer $(10 \mathrm{mmol} / \mathrm{L} \mathrm{pH} 6.0)$ by water-bath kettle heating for $30 \mathrm{~min}$. Endogenous peroxidase was blocked in $0.3 \%$ hydrogen peroxide for $10 \mathrm{~min}$. Nonspecific binding was blocked by incubation in $10 \%$ normal animal serum for $10 \mathrm{~min}$. Sections were incubated at $4^{\circ} \mathrm{C}$ for $24 \mathrm{~h}$ with primary antibodies including polyclonal antibody against anti-CPA4 (Sigma-Aldrich), anti-Survivin and anti-VEGF (Santa Cruz Inc., USA). Protein expression levels were scored by staining intensity and the percentage of immunoreactive cancer cells. Tissues with no staining were rated as 0 , with faint staining or moderate to strong staining in $10 \%$ of cells as 1 , with moderate staining or strong staining in $10 \%$ to $50 \%$ of cells as 2 , and with strong staining in $>50 \%$ of cells as 3 . Lung cancer tissues that registered levels 0 and 1 were defined as negative for expression, whereas samples at levels 2 or 3 were defined as positive.

\section{Measurement of Human Plasma Levels of CPA4 VEGF and Survivin}

For ELISA study, blood samples were obtained from 100 lung cancer patients (median age at 58 with a range of 31 to 79 years) during 2007-2008 including 52 lung squamous cancer samples and 48 lung adenocarcinoma samples. 80 specimens of healthy individuals with similar age (median age at 56 with a range of 36 to 81 years) and sex distribution were analyzed, which were donated on a voluntary basis. For all the specimens, clinical data were obtained retrospectively for all lung cancer patients, including age, gender, differentiation, and TNM stage. The study was approved by the medical ethics committee of Cancer Institute and Hospital, CAMS. CPA4 (SEF317Hu, Cloud-Clone Corp.), Survivin (Quantikine Human survivin Immunoassay, R\&D System, USA), and VEGF (Quantikine Human VEGF Immunoassay, R\&D System, USA) concentrations were determined by means of an enzyme linked immunosorbent assay (ELISA) method according to the manufacturer's instructions. The range of the immunoassays for CPA4, Survivin and VEGF were 0.156 to $10 \mathrm{ng} / \mathrm{mL}, 1.58$ to $9.96 \mathrm{pg} / \mathrm{ml}$, and 31.20 to $2,000 \mathrm{pg} / \mathrm{mL}$, respectively. Each serum sample was run in duplicate. Briefly, $100 \mathrm{ml}$ of serum (1:5 dilution) were placed into each well of the ELISA plate and 
incubated for $45 \mathrm{~min}$ at $37^{\circ} \mathrm{C}$. The plates were washed four times with buffer and incubated with $100 \mathrm{ml}$ of detection antibody at $37^{\circ} \mathrm{C}$ for $45 \mathrm{~min}$. After four washes, the plates were incubated with substrate solution for $15 \mathrm{~min}$ at room temperature, then the reaction was stopped and the plates were read by a spectrophotometer at wavelength $450 \mathrm{~nm}$. A standard curve was generated with the provided standards and used to calculate the quantity of CPA4 in each serum sample.

\section{Statistical Analysis}

The SPSS 15 software package (SPSS, Inc., Chicago, IL) was used for statistical analysis. The independent sample $t$ test was used for ELISA group analysis. The association between the markers and clinicopathologic features was analyzed using $X^{2}$-test or two-sided t-test as appropriate. Kaplan-Meier analysis was adopted to evaluate the effects of CPA4 expression on the overall survival (OS) of patients with lung cancer. Whether CPA4 could serve as a prognostic marker in lung cancer was estimated by Cox-regression analysis. Logistic regressions were used to assess association between CPA4 overexpression and NSCLC. Receiver Operating Characteristic (ROC) curves of biomarkers levels were also calculated. All comparisons were two-tailed, and p-value of $<0.05$ was considered significant.

\section{Results}

\section{CPA4 is overexpressed in lung cancer at DNA and $m R N A$ level}

To roundly investigate CPA4 expression in lung cancer tissues, we analyzed three independent datasets from Oncomine. The results showed that CPA4 gene copy number was increased in lung cancer tissues in comparison to normal lung tissues in TCGA and Weiss Lung datasets (Figure 1A). And, we also observed the upregulation of the CPA4 mRNA level in lung cancer tissues compared with normal tissues in Hou lung dataset (Figure 1B). These observations suggested that CPA4 was highly expressed in lung cancer tissues especially in NSCLC.

\section{CPA4 expression in NSCLC tissues by immunohistochemistry}

To examine the clinical relevance of CPA4 in human NSCLC, we evaluated its levels and distribution in Human NSCLC tissue arrays by immunohistochemistry. The results showed that 120 of 165 of the primary lesions exhibited positive staining for CPA4, but there was no staining of adjacent normal tissues (Figure 2A). Furthermore, CPA4 positive staining was observed in cancer cells as well as interstitial tissues especially around micro-vessels areas in lung cancer tissues. Then, we detected tumor-related angiogenesis factors including vascular endothelial growth factor (VEGF) and Survivin in the same type of tissue arrays. The results demonstrated that the positive rates of Survivin and VEGF were $57.6 \%(95 / 165)$ and $49.7 \%$ (82/165), respectively (Figure 2B, 2C). Statistical analysis revealed that the levels of $\mathrm{CPA} 4$ were significantly correlated with Tumor size $(\mathrm{P}=0.005)$, Depth of invasion $(\mathrm{P}=0.008)$, Lymph Node Metastasis $(\mathrm{P}=0.001)$, Stage $(\mathrm{P}=0.001)$, VEGF $(\mathrm{P}=0.037)$ and Survivin level $(\mathrm{P}=0.049)$.
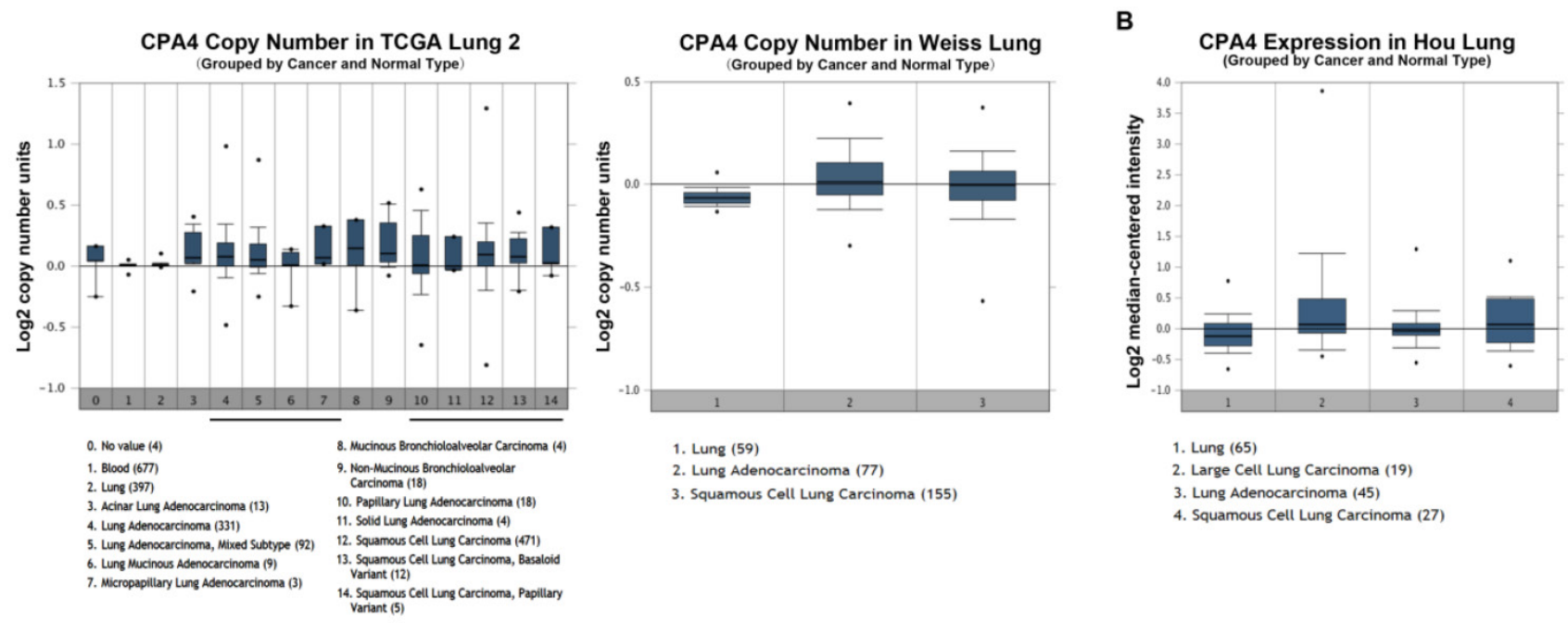

Figure 1. CPA4 mRNA or DNA expression in human lung cancers using the Oncomine database. A. CPA4 gene copy number in human lung cancers vs. normal tissues. B. CPA4 mRNA expression in human lung cancers vs. normal tissues. 
A
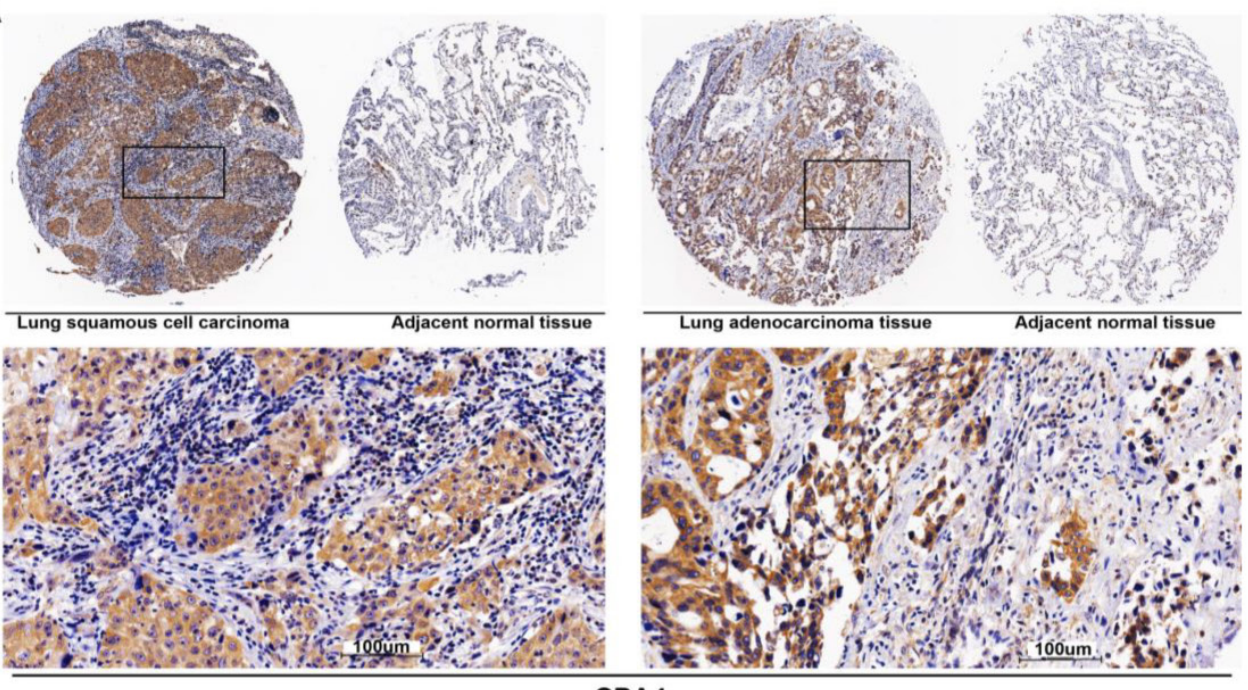

CPA4

B
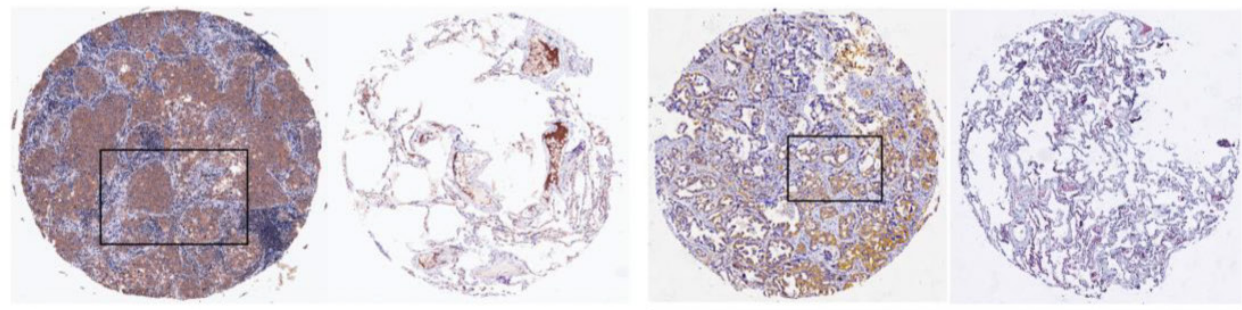

Lung squamous cell carcinoma $\quad$ Adjacent normal tissue
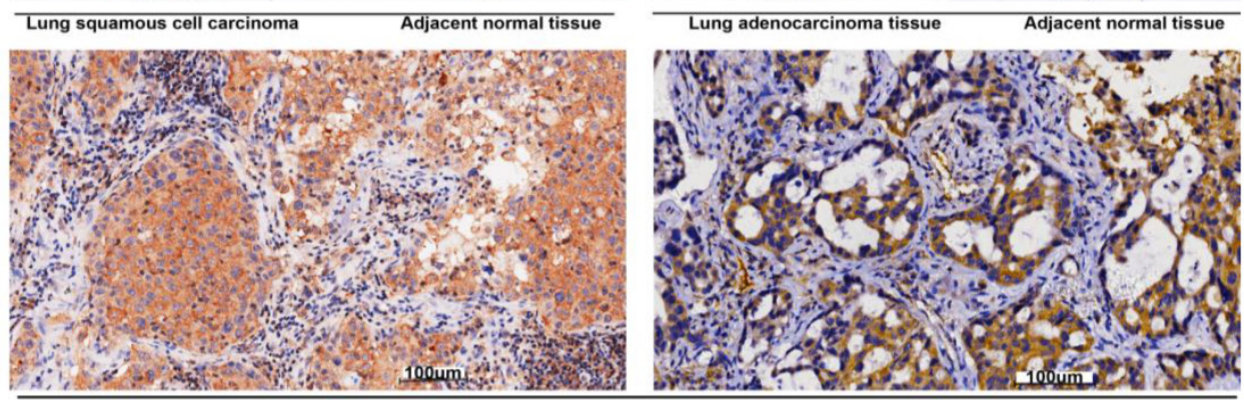

VEGF

C
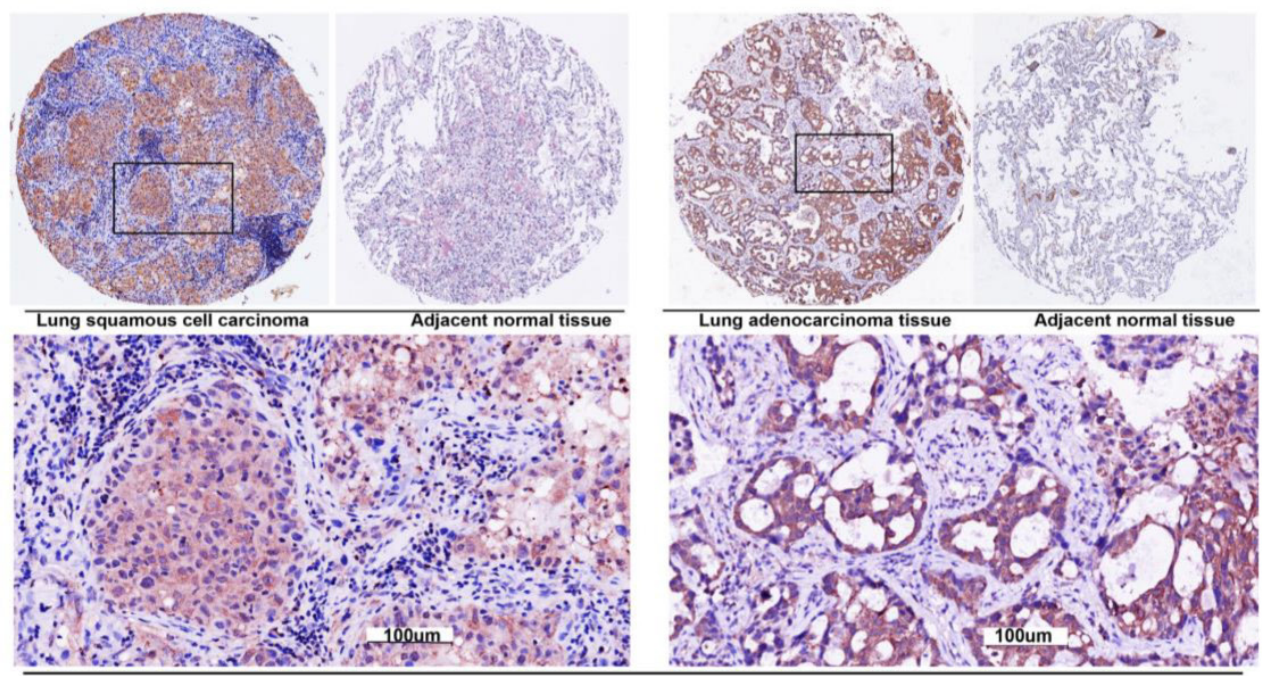

Survivin

Figure 2. CPA4, Survivin and VEGF expression in lung cancer tissues were determined by immunochemistry. A. Positive expression of CPA4, B. Positive expression of VEGF, and C. Positive expression of Survivin. 
However, no statistically significant correlations were identified between the expression of CPA4 and other clinicopathologic characteristics including age, gender, grade and distant metastasis (Table 1). Furthermore, Pearson correlation analysis demonstrated that the expression of CPA4 was closely associated the level of Survivin $(\mathrm{r}=0.398, \mathrm{p}=0.000)$ and VEGF ( $\mathrm{r}=0.258, \mathrm{P}=0.001)$ (Table 2).

Table 1. Correlation between CPA4 expression and clinicopathological characteristics in 165 lung cancer cases.

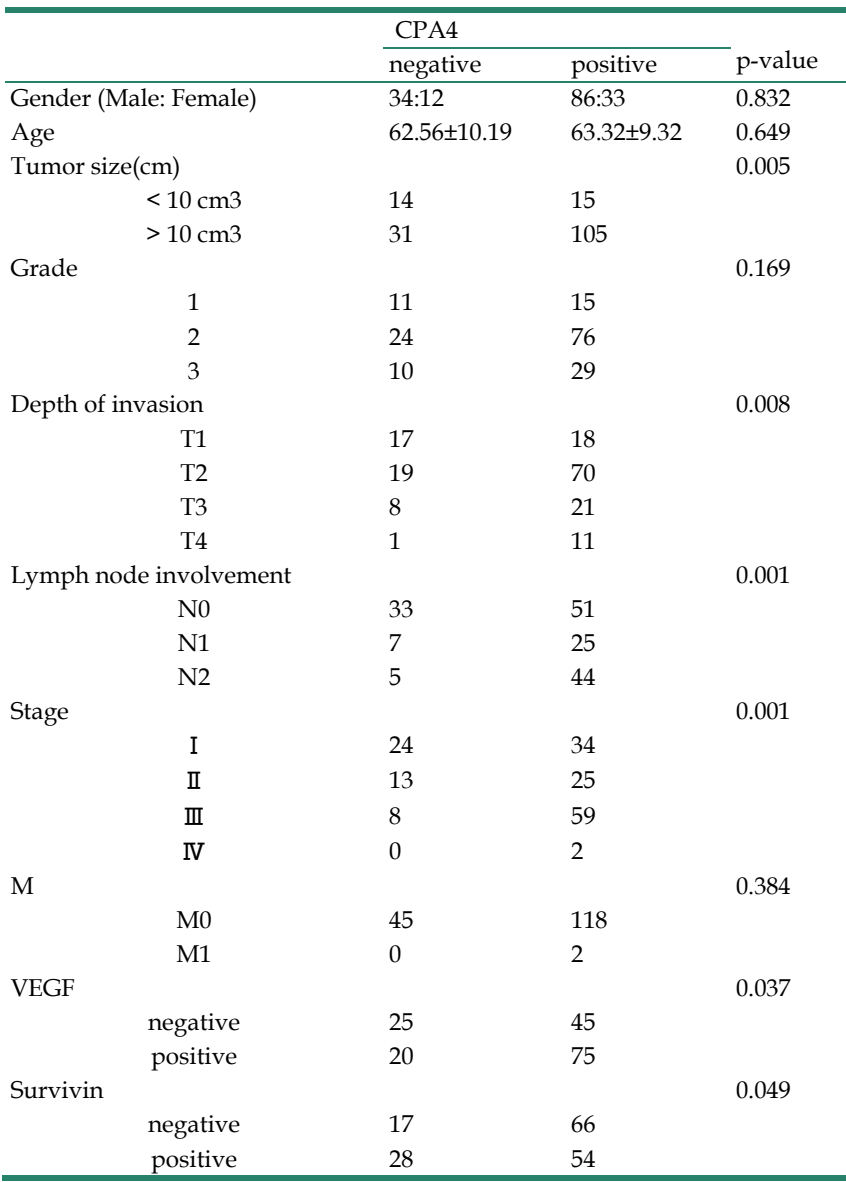

Table 2. Correlations between the expression of CPA4, VEGF and Survivin.

\begin{tabular}{llll}
\hline & & Survivin & VEGF \\
\hline \multirow{3}{*}{ CPA4 } & Pearson Correlation & $0.398^{* *}$ & $0.258^{* *}$ \\
& Sig. (2-tailed) & 0.000 & 0.001 \\
& $\mathrm{~N}$ & 165 & 165 \\
\hline
\end{tabular}

**. Correlation is significant at the 0.01 level (2-tailed). *. Correlation is significant at the 0.05 level (2-tailed)

\section{Overexpression of CPA4 correlates with poor prognosis in NSCLC}

To determine the correlation between CPA4 expression and clinical outcome, we analyzed the prognostic significance of CPA4 using Kaplan-Meier analysis.
As shown in Figure 3A, the overall survival time of lung cancer patients with CPA4-positive expression was markedly shorter than those with CPA4-negative expression (Figure 3A). Similarly, we found that patients with high Survivin or VEGF expression had a poor prognosis, respectively (Figure 3B, 3C). Moreover, the subsequent univariate Cox regression model demonstrated that CPA4 level ( $\mathrm{HR}=1.832 ; 95 \%$ CI: 1.055-3.179; $\mathrm{P}=0.031$ ) and lymph node metastasis $(\mathrm{HR}=2.075 ; \quad 95 \% \quad \mathrm{CI}: 1.122-3.836 ; \quad \mathrm{P}=0.020)$ were statistically independent predictive factors of poorer prognosis for lung cancer (Table 3 ).

Table 3. Multivariate analysis of Cox Proportional Hazards Model for lung cancer.

\begin{tabular}{|c|c|c|c|c|c|c|c|c|}
\hline \multirow[t]{2}{*}{ Characteristics } & \multirow[t]{2}{*}{ B } & \multirow[t]{2}{*}{ SE } & \multirow[t]{2}{*}{ Wald } & \multirow[t]{2}{*}{ df } & \multirow[t]{2}{*}{ Sig. } & \multirow[t]{2}{*}{$\operatorname{Exp}(B)$} & \multicolumn{2}{|c|}{$\begin{array}{l}95.0 \% \text { CI for } \\
\operatorname{Exp}(B)\end{array}$} \\
\hline & & & & & & & Lower & Upper \\
\hline CPA4 level & .605 & .281 & 4.629 & 1 & .031 & 1.832 & 1.055 & 3.179 \\
\hline $\begin{array}{l}\text { Tumor Size } \\
(\mathrm{cm} 3)\end{array}$ & -.156 & .311 & .251 & 1 & .616 & .856 & .465 & 1.574 \\
\hline $\begin{array}{l}\text { Depth of } \\
\text { invasion }\end{array}$ & .511 & .345 & 2.194 & 1 & .139 & 1.667 & .848 & 3.278 \\
\hline $\begin{array}{l}\text { Lymph node } \\
\text { metastasis }\end{array}$ & .730 & .314 & 5.421 & 1 & .020 & 2.075 & 1.122 & 3.836 \\
\hline Stage & -.076 & .364 & .043 & 1 & .835 & .927 & .454 & 1.892 \\
\hline VEGF level & -.160 & .210 & .578 & 1 & .447 & .852 & .564 & 1.287 \\
\hline Survivin level & -.179 & .218 & .675 & 1 & .411 & .836 & .546 & 1.281 \\
\hline
\end{tabular}

\section{Elevated Serum Levels of CPA4 in NSCLC Patients}

To investigate the potential of CPA4 as a serological marker for NSCLC, we analyzed the level of CPA4 in sera from NSCLC patients $(n=100)$ and healthy controls $(n=80)$ by ELISA. The result demonstrated that the serum levels of CPA4 in patients with NSCLC were significantly higher than those of the healthy group (CPA4: 2915 \pm 747 vs $2170 \pm 767 \mathrm{pg} / \mathrm{mL}, \quad \mathrm{P}=0.000$ ) (Figure 4A). When classified according to histologic type of lung cancer, the serum levels of CPA4 were $2961 \pm 814 \mathrm{pg} / \mathrm{mL}$ in lung squamous cell carcinoma (LSCC, $n=52$ ), and $2.866 \pm 0.672$ in lung Adenocarcinoma $(\mathrm{AD}, \mathrm{n}=48)$, which were also higher than normal ones (Figure 4B).

\section{Clinical significance of CPA4 serum level in NSCLC}

ROC curves for serum CPA4 concentrations were constructed to determine the cutoff values. The approximate area under the ROC curve assessing serum CPA4 as a diagnostic tool for the detection of lung cancer against normal controls was 0.781 , at a cutoff value of $2700 \mathrm{pg} / \mathrm{ml}$. (Fig. 4C). Then, we analyzed the association between serum CPA4 level above the cutoff level and clinicopathological 
parameters. As demonstrated in Table 4, the concentrations of CPA4 were significantly correlated with Lymph node involvement $(\mathrm{p}=0.038)$ and differentiation $(p=0.041)$. Thus, the elevation of serum CPA4 levels appears to be closely associated with lung cancer progression.

\section{The diagnostic value of CPA4 as a serologic biomarker for NSCLC}

To further evaluate the potential clinical utility of CPA4 only or combining with other indicators as the diagnostic serum markers, we employed the ROC curve analysis to assess other five common serum markers (VEGF, Survivin, NSE, CYFRA21-1 and SCCA) to discriminate between NSCLC patients and healthy volunteers. As shown in Figure 4D, the AUCs of VEGF, Survivin, NSE, CYFRA21-1 and SCCA were $0.531 \quad(95 \% \quad$ CI: $\quad 0.445-0.618), \quad 0.368 \quad(95 \% \quad$ CI: $0.287-0.450), 0.288$ (95\% CI: $0.214-0.362), 0.607$ (95\% CI: $0.522-0.692$ ) and 0.493 (95\% CI: 0.408-0.578), respectively. To further improve diagnostic accuracy, Logistic regression was used to calculate the respective significance of each candidate marker for NSCLC diagnosis. The results indicated that CPA4 and CYFRA21-1 showed diagnostic performance $(\mathrm{P}<0.05)$, and the odds ratios were 14.823 and 4.16, respectively (Table 5). Then, the logistic regression equation was built: $\operatorname{logitP}=-1.776+2.696^{*} \mathrm{CPA} 4+1.425^{*}$ CYFRA21-1, where the levels of CPA4 and CYFRA21-1 in serum were standardized for normalized value. According to the discriminant equation, " 0 " was defined as the cut point. Using the two biomarkers, the diagnostic assay can effectively discriminate NSCLC patients from normal controls.
The sensitivity and specificity were $86 \%$ and $70 \%$, respectively. Moreover, the combination had an AUC-ROC $=0.830$.

Table 4. Correlation between CPA4 level in NSCLC serum.

\begin{tabular}{llll}
\hline \multicolumn{3}{l}{ Serum CPA4 } & \\
\hline & negative & positive & p value \\
Sex (Male: Female) & $19: 12$ & $53: 16$ & 0.110 \\
Age & $59.84 \pm 10.68$ & $57.28 \pm 8.06$ & 0.188 \\
Differentiation & & & 0.041 \\
high & 18 & 25 & \\
moderate+low & 13 & 44 & \\
& & & \\
Depth of invasion & & & 0.249 \\
T1 & 7 & 14 & \\
T2 & 7 & 29 & \\
T3 & 9 & 16 & \\
T4 & 8 & 10 & \\
Lymph node involvement & & & 0.038 \\
N0 & 7 & 12 & \\
N1 & 12 & 11 & \\
N2 & 12 & 44 & \\
N3 & 0 & 2 & \\
Distant Metastasis & & & \\
M0 & 29 & 61 & \\
M1 & 2 & 8 & \\
\hline
\end{tabular}

Table 5. Logistic regression analysis of 6 serum markers.

\begin{tabular}{lllllllll}
\hline & \multicolumn{1}{c}{} & \multicolumn{1}{c}{} & \multicolumn{1}{c}{} & \multicolumn{3}{c}{$\begin{array}{c}\text { 95\% C.I.for } \\
\text { EXP(B) }\end{array}$} \\
\hline & B & S.E. & Wald & df & Sig. & Exp(B) & Lower & Upper \\
\hline VEGF & 0.544 & 0.415 & 1.718 & 1 & 0.190 & 1.722 & 0.764 & 3.884 \\
Survivin & 0.013 & 0.52 & 0.001 & 1 & 0.980 & 1.013 & 0.366 & 2.804 \\
NSE & -1.012 & 0.503 & 4.043 & 1 & 0.044 & 0.363 & 0.136 & 0.975 \\
CYFRA21 & 1.425 & 0.431 & 10.921 & 1 & 0.001 & 4.16 & 1.786 & 9.688 \\
SCCA1 & -0.328 & 0.435 & 0.57 & 1 & 0.450 & 0.72 & 0.307 & 1.688 \\
CPA4 & 2.696 & 0.439 & 37.804 & 1 & 0.000 & 14.823 & 6.276 & 35.012 \\
Constant & -1.776 & 0.508 & 12.213 & 1 & 0.000 & 0.169 & & \\
\hline
\end{tabular}
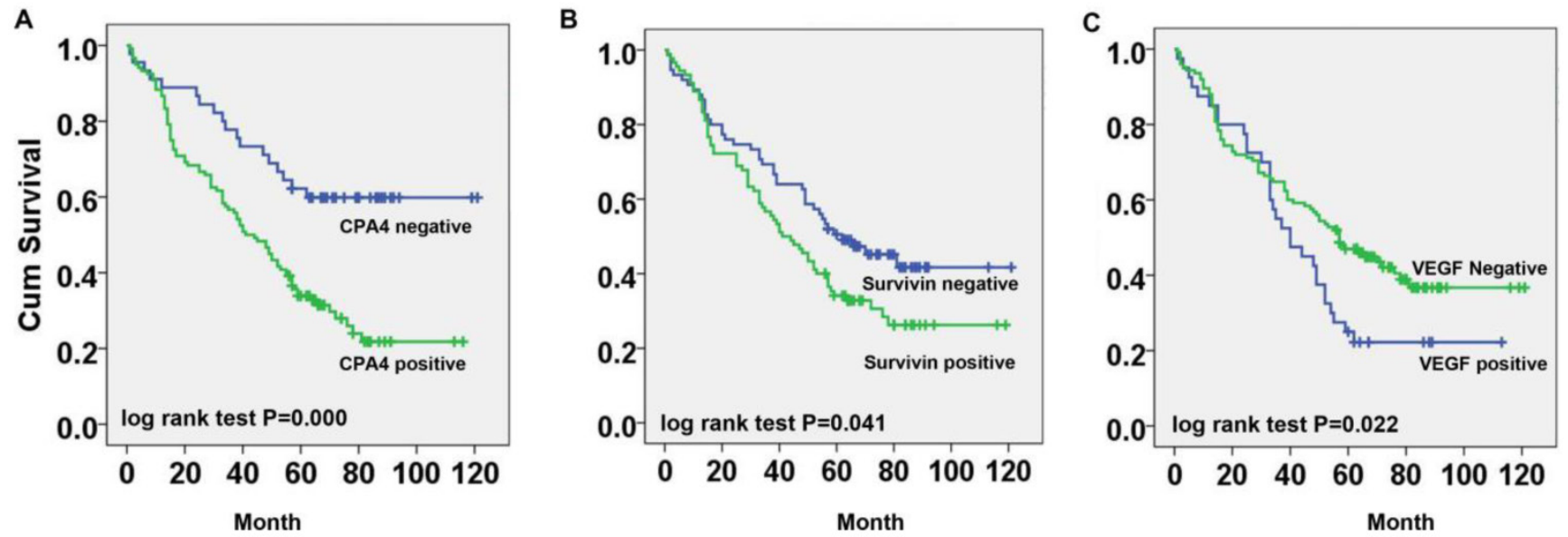

Figure 3. Survival curves for NSCLC cancer using the Kaplan-Meier method and the log-rank test. A. Overall survival curves for patients with negative CPA4 expression (blue line) and patients with positive CPA4 (green line); B. Overall survival curves for patients with negative Survivin expression (blue line) and patients with positive Survivin (green line); C. Overall survival curves for patients with negative VEGF expression (green line) and patients with positive VEGF (blue line). 
A

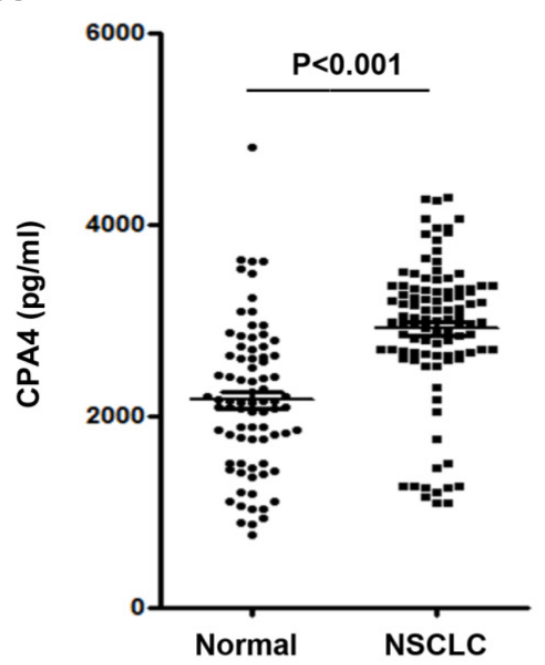

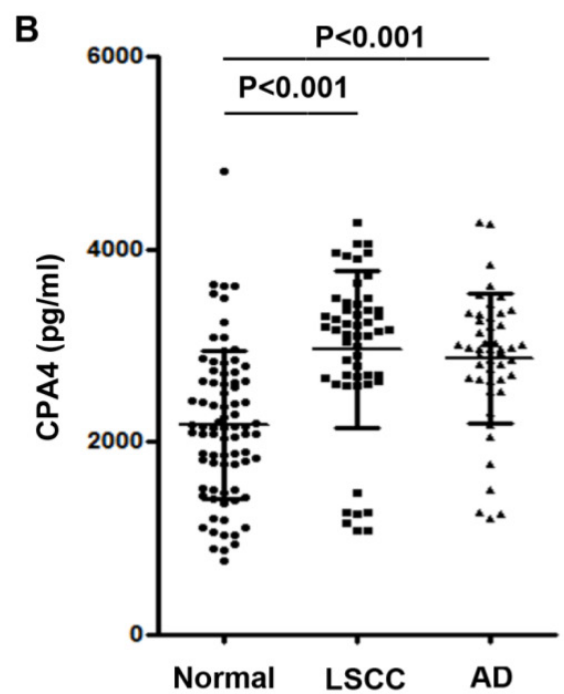

LSCC AD
C

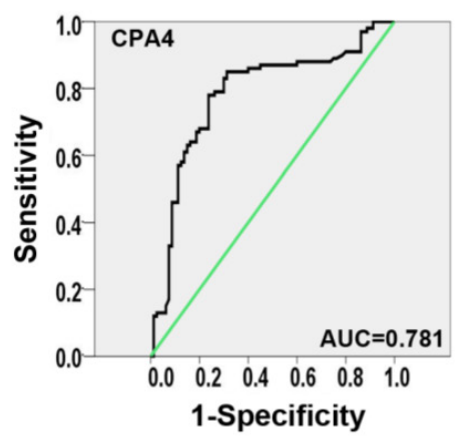

D

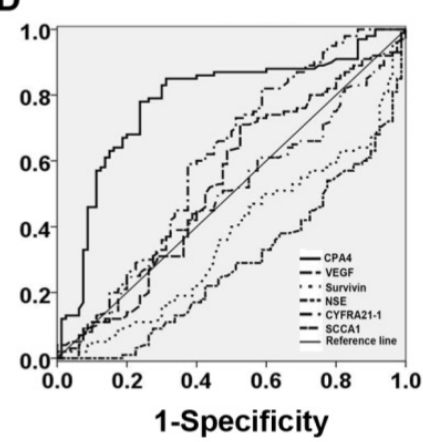

E

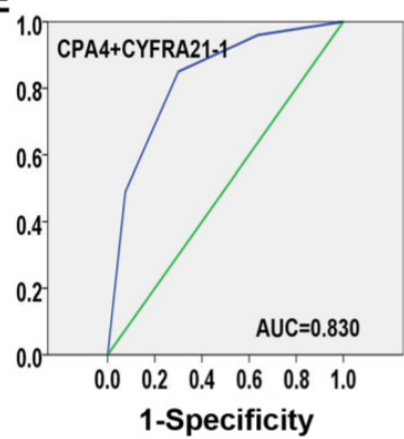

Figure 4. Elevated Serum CPA4 levels in lung cancer serum samples. A. Serum levels of CPA4 in healthy controls $(n=80)$ and lung cancer patients $(n=100)$. B. Serum levels of CPA4 in healthy controls $(n=80)$, lung squamous cell carcinomas $(L S C C, n=52)$ and $A d e n o c a r c i n o m a(A D, n=48)$. C. Receiver operator curves demonstrating the ability of CPA4. D. Receiver operator curves demonstrating the ability of serum CPA4, CYFRA21-1, NSE, SCCA, VEGF and Survivin alone to predict lung cancer. E. Receiver operator curves demonstrating the ability of the combination between CPA4 and CYFRA21-1.

\section{Discussion}

Lung cancer is a leading cause of cancer death worldwide, and early diagnosis can greatly improve cancer survival rates [12]. Serum tumor markers including neuron specific enolase (NSE), cytokeratin fragment (CYFRA21-1) and squamous carcinoma antigen (SCCA) has been used in the diagnosis of lung cancer $[4,13]$. However, the diagnostic effectiveness of a single marker is limited by its low specificity and/or sensitivity. Therefore, there is an urgent need to find out other potential biomarkers in clinical practice.

Recent studies indicated that tumor cells can release factors that facilitate angiogenesis and create unique tumor microenvironment [14]. Moreover, these secreted protein could be measured as cancer markers in early detection of cancers $[15,16]$. Carboxypeptidase A4 (CPA4) is a secreted protein with metallocarboxypeptidase activity [9]. In prostate cancer, CPA4 gene is imprinted and may become a strong candidate gene for prostate canceraggressiveness [17]. Our previous study also indicated that CPA4 level was significantly elevated in pancreatic cancer tissues as well as serum samples, and was closely associated with tumor progression.

Despite the potential implication of CPA4 for the cancer progression, no previous studies have examined its level and clinical significance in lung cancer. In this study, we firstly demonstrated that CPA4 was overexpressed in NSCLC tissues in both RNA and DNA level by using the publicly available gene expression datasets in the Oncomine. Then, we evaluated the CPA4 level in human NSCLC tissue arrays by using IHC. We found that CPA4 was highly expressed in NSCLC cancer tissues, with a positive rate of $72.7 \%$. We also observed that CPA4 positive staining was observed in cancer cells as well as stromal compartment especially around micro-vessels areas in lung cancer tissues. It has been reported that angiogenesis-related factors including VEGF and Survivin were involved in neo-angiogenesis [18], 
which were significantly correlated with poor prognosis in certain types of cancers, such as NSCLC [19], ovarian carcinoma [20] and thyroid carcinoma [21]. Then, we detected their levels in the same type of tissue arrays, and found that overexpression of CPA4 was significantly associated with the high level of Survivin or VEGF.

Clinical relevance analysis showed that positive expression of CPA4 in NSCLC tissues was significantly correlated with Tumor size, Depth of invasion, Lymph Node Metastasis and Stage. Moreover, high CPA4 expression was significantly associated with for NSCLC patients, as an independent prognostic factor.

CPA4 is a secreted protein and highly expressed in lung cancer tissues, so we tested whether serum level of CPA4 could be used as a tumor marker for NSCLC.

Our results demonstrated that CPA4 serum levels in the NSCLC patients were much higher than that in healthy controls. Furthermore, we showed that the serum level of CPA4 was significantly correlated with Lymph node involvement $(\mathrm{p}=0.038)$ and differentiation $(p=0.041)$ and could be an independent prognostic factor.

Then, we evaluated the value of CPA4 serum level for the early diagnosis of lung cancer. Because the diagnostic effectiveness of a single marker is limited, we also analyzed the most commonly investigated tumor markers including NSE, CYFRA21-1 and SCCA individually or in combination $[22,23]$. ROC curve analysis revealed that the accuracy of serum CPA4 and CYFRA21-1 for the diagnosis of NSCLC was superior to that of NSE, SCCA, Survivin and VEGF.

We also found that combinations of CPA4 and CYFRA21-1 can improve diagnostic efficacy compared with a single marker, and area under the ROC curve (AUC) was increased to 0.830 .

In conclusion, our study demonstrated that CPA4 overexpression in NSCLC tissues is significantly associated with poor survival. CPA4 can be used as a novel diagnostic and prognostic serum marker for human non-small-cell lung cancer, and serum CPA4 level combining with serum CYFRA21-1 level could be used to aid early detection of NSCLC.

\section{Acknowledgement}

Supported by grant from Beijing Nova Program (No: Z1511000003150121), National High-tech R\&D Program of China for Young Scholars (No: 2014AA020537).

\section{Competing Interests}

No potential conflicts of interest were disclosed.

\section{References}

1. Islami F, Torre LA, Jemal A. Global trends of lung cancer mortality and smoking prevalence. Translational Lung Cancer Research. 2015; 4: 327-38.

2. Billmeier SE, Ayanian JZ, Zaslavsky AM, Nerenz DR, Jaklitsch MT, Rogers SO. Predictors and Outcomes of Limited Resection for Early-Stage Non-Small Cell Lung Cancer. Journal of the National Cancer Institute. 2011; 103: 1621-9.

3. Holdenrieder S, von Pawel J, Dankelmann E, Duell T, Faderl B, Markus A, et al. Nucleosomes, ProGRP, NSE, CYFRA 21-1, and CEA in Monitoring First-Line Chemotherapy of Small Cell Lung Cancer. Clinical Cancer Research. 2008; 14: 7813-21

4. Schneider J, Philipp M, Velcovsky HG, Morr H, Katz N. Pro-gastrin-releasing peptide (ProGRP), neuron specific enolase (NSE), carcinoembryonic antigen (CEA) and cytokeratin 19-fragments (CYFRA 21-1) in patients with lung cancer in comparison to other lung diseases. Anticancer research. 2003; 23: 885-93.

5. Lee JH, Chang JH. Diagnostic utility of serum and pleural fluid carcinoembryonic antigen, neuron-specific enolase, and cytokeratin 19 fragments in patients with effusions from primary lung cancer. Chest. 2005; 128: 2298-303

6. Weis SM, Cheresh DA. Tumor angiogenesis: molecular pathways and therapeutic targets. Nature medicine. 2011; 17: 1359-70.

7. Plate KH, Breier G, Weich HA, Risau W. Vascular endothelial growth factor is a potential tumour angiogenesis factor in human gliomas in vivo. Nature. 1992; 359: 845-8

8. Virrey JJ, Guan S, Li W, Schonthal AH, Chen TC, Hofman FM. Increased survivin expression confers chemoresistance to tumor-associated endothelial cells. The American journal of pathology. 2008; 173: 575-85.

9. Tanco S, Zhang X, Morano C, Avilés FX, Lorenzo J, Fricker LD. Characterization of the Substrate Specificity of Human Carboxypeptidase A4 and Implications for a Role in Extracellular Peptide Processing. Journal of Biological Chemistry. 2010; 285: 18385-96.

10. Tanco S, Zhang X, Morano C, Aviles FX, Lorenzo J, Fricker LD. Characterization of the substrate specificity of human carboxypeptidase A4 and implications for a role in extracellular peptide processing. J Biol Chem. 2010; 285: 18385-96.

11. Chen L, Wei T, Si X, Wang O, Li Y, Leng $Y$, et al. Lysine Acetyltransferase GCN5 Potentiates the Growth of Non-small Cell Lung Cancer via Promotion of E2F1, Cyclin D1, and Cyclin E1 Expression. The Journal of Biological Chemistry. 2013; 288: 14510-21.

12. DeSantis CE, Lin CC, Mariotto AB, Siegel RL, Stein KD, Kramer JL, et al. Cancer treatment and survivorship statistics, 2014. CA Cancer J Clin. 2014; 64: 252-71.

13. Shibayama T, Ueoka H, Nishii K, Kiura K, Tabata M, Miyatake $K$, et al. Complementary roles of pro-gastrin-releasing peptide (ProGRP) and neuron specific enolase (NSE) in diagnosis and prognosis of small-cell lung cancer (SCLC). Lung cancer. 2001; 32: 61-9.

14. Samples J, Willis M, Klauber-DeMore N. Targeting Angiogenesis and the Tumor Microenvironment. Surgical oncology clinics of North America. 2013; 22: 629-39.

15. Aikou S, Ohmoto Y, Gunji T, Matsuhashi N, Ohtsu H, Miura H, et al. Tests for Serum Levels of Trefoil Factor Family Proteins Can Improve Gastric Cancer Screening. Gastroenterology. 2011; 141: 837-45.e7.

16. Shin J, Kim H-J, Kim G Song M, Woo SJ, Lee S-T, et al. Discovery of Melanotransferrin as a Serological Marker of Colorectal Cancer by Secretome Analysis and Quantitative Proteomics. Journal of Proteome Research. 2014; 13: 4919-31.

17. Kayashima T, Yamasaki K, Yamada T, Sakai H, Miwa N, Ohta T, et al. The novel imprinted carboxypeptidase A4 gene ( CPA4) in the 7q32 imprinting domain. Hum Genet. 2003; 112: 220-6.

18. Tran J, Master Z, Yu JL, Rak J, Dumont DJ, Kerbel RS. A role for survivin in chemoresistance of endothelial cells mediated by VEGF. Proceedings of the National Academy of Sciences of the United States of America. 2002; 99: 4349-54.

19. Wang M, Liu BG, Yang ZY, Hong X, Chen GY. Significance of survivin expression: Prognostic value and survival in stage III non-small cell lung cancer. Experimental and therapeutic medicine. 2012; 3: 983-8.

20. Dobrzycka B, Mackowiak-Matejczyk B, Terlikowska KM, Kulesza-Bronczyk B, Kinalski M, Terlikowski SJ. Prognostic significance of pretreatment VEGF, survivin, and Smac/DIABLO serum levels in patients with serous ovarian carcinoma. Tumour Biology. 2015; 36: 4157-65.

21. Zhang H-Y, Meng X, Du Z-X, Fang C-Q, Liu G-L, Wang H-Q, et al. Significance of survivin, caspase-3, and VEGF expression in thyroid carcinoma. Clinical and Experimental Medicine. 2009; 9: 207-13.

22. Okamura $K$, Takayama K, Izumi M, Harada T, Furuyama K, Nakanishi $Y$. Diagnostic value of CEA and CYFRA 21-1 tumor markers in primary lung cancer. Lung cancer. 2013; 80: 45-9.

23. Matsuoka K, Sumitomo S, Nakashima N, Nakajima D, Misaki N. Prognostic value of carcinoembryonic antigen and CYFRA21-1 in patients with pathological stage I non-small cell lung cancer. European Journal of Cardio-Thoracic Surgery. 2007; 32: 435-9. 